\title{
Procedure for Experimental Data Assessment for Numerical Solver Validation in the Context of Model based Prediction of Powder Coating Patterns
}

\author{
B Siyahhan', M Boldrini', S Hauri², N Reinke ${ }^{2}$, \\ G Boiger ${ }^{1 *}$ \\ 1. ZHAW, Switzerland \\ 2. Winterthur Instruments, Switzerland
}

\begin{abstract}
In the scope of this study an experimental powder coating setup is designed and the method to extract statistically significant trends from the data generated is developed. The ultimate goals are to i) validate a previously developed 3D Euler-LaGrangian numerical solver [1] and to ii) characterize the essential parameters for industrial powder coating processes in subsequent phases. The experiments involved coating a flat plate substrate with a corona spraying pistol. The resulting coating thickness has been quantified through the state of the art Coatmaster technology [2,3]. The raw data generated from the Coatmaster has been filtered and rigorously analyzed to identify statistically significant trends. Furthermore, characteristic variables have been constructed for subsequent comparison to the numerical solver. This study reveals the challenges involved in assessing experimental data to extract meaningful comparisons for numerical solver validation.
\end{abstract}

\section{INTRODUCTION}

Powder coating is an environmentally friendly alternative to other methods for providing surface finishes, having a wide range of application areas such as the automotive-, construction- and household appliance industries amongst others [4]. Powder coating is applied electrostatically where a particle laden flow passes through a corona within a spraying pistol, whereby the particles are charged, subsequently coating the grounded substrate. Hence from a physical point of view there are several phenomena at play [17]; the turbulent air flow $\left(\operatorname{Re}_{\max } \approx 1.5 \mathrm{e} 5, \mathrm{U}_{\max } \approx 20 \mathrm{~m} / \mathrm{s}\right)$, carrying polymer particles $(2 \mu \mathrm{m}-180 \mu \mathrm{m})$ that interact with each other, the overlaid electrostatic field $\left(\psi_{\max } \leq 1.2 \mathrm{e} 5 \mathrm{~V}\right)$ and the gravitational field. The complex interactions involved are hard to investigate experimentally as well as numerically, therefore most development in the field has relied on empirical methods [5]. In order to develop a systematic approach to process parameter optimization or technology enhancement through new designs, a validated numerical tool is essential, which in turn requires a comprehensive experimental study of the coating process. Some of the experimental studies published to date focus on details of the particle velocity and size distribution [6] or electrostatic properties of the powder [7]. Others that attempt to quantify the coating efficiency [8-10], use destructive

${ }^{*}$ Corresponding Author: boig@zhaw.ch 
methods and do not provide information on the distribution of the particles on the substrate. As a result, the numerical investigations either lack an experimental comparison [11] or are based on the velocity field distribution [12], and indirect derivation of the film thickness from it [13]. In practice however, it is essential to assess the coating distribution on a substrate as well as its thickness. Hence a non-invasive method to gather this information is crucial, as well as quantification through the post-processing of the data. This assessment procedure would form the basis of a validation process for a numerical solver that can then be applied to optimize industrial coating processes and aid in further technology development. To this end, a novel method to gather the coating thickness values and distributions on substrates is introduced in this paper. Also, a special emphasis is afforded to the description of the filtering procedure applied to the data gathered, as well as the quantification of the key parameters for the coating process.

\section{MATERIAL AND METHOD}

\subsection{Experimental Set-up}

The experimental set-up consists of a corona type spraying gun, that is fixed within the coating chamber, to which a plastic railing is mounted, that also supports the holder for the $10 \mathrm{~cm}$ by $10 \mathrm{~cm}$ grounded plate substrate as can be seen in Figure 1. An air draft is generated by a ventilation system at the filtration site to collect the residual powder.

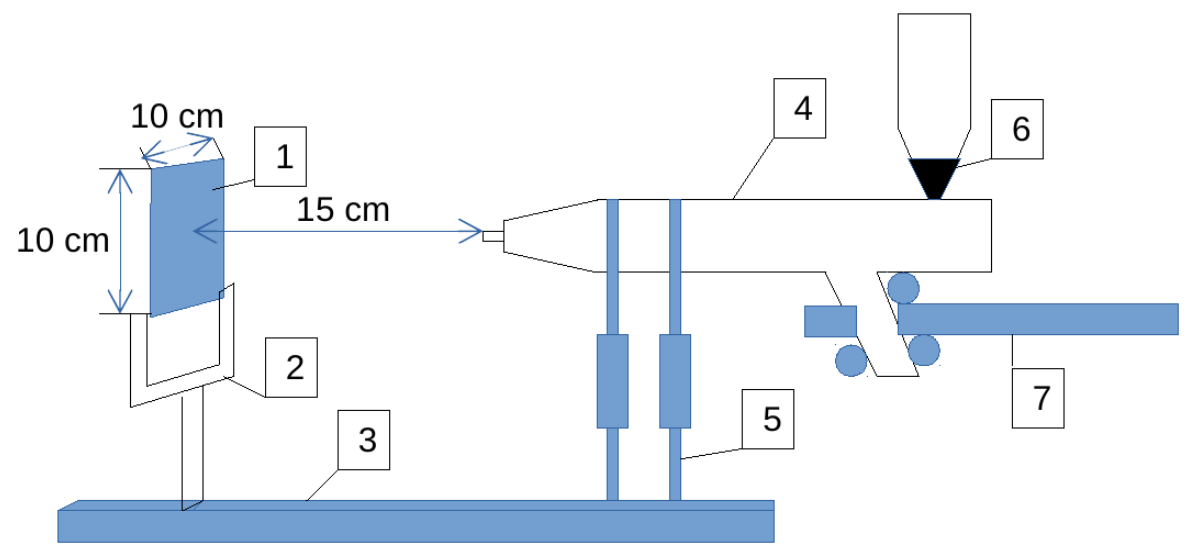

Figure 1- The experimental setup. Substrate (1) is fixed with the aid of the clamp holder (2) to the common railing (3) onto which also the coating pistol (4) is mounted (5). The pistol contains $5 \mathrm{gr}$ of powder (6) and is aligned with the center of the substrate with the roller holder (7) on the back side.

The experiments are carried out with a fixed amount of $5 \mathrm{gr}$ of powder at varying voltages where the substrates are placed at a distance of $15 \mathrm{~cm}$ from the pistol. Once a substrate is coated with a certain set of parameters, the thickness of the coating is measured using the Coatmaster technology. The measurements are carried out three times at the front- and three times at the back side, allowing for a recovery time between each measurement. Additionally, three plates are coated for each voltage setting, resulting in nine measurements per setting so that statistical analysis can be performed. 


\subsection{Thickness Measurement with Coatmaster 3D}

Coatmaster 3D allows the thickness of a coating to be measured without contact, right after the application (uncured coatings), within a range of $100 \mathrm{~cm}$. The technology is based on advanced thermal optics, where the coating layer to be measured is heated in a pulsed manner by a computer controlled light source. Then a high-speed infrared detector records the temperature profile in a time series from a distance. As the surface temperature diminishes, depending on the coating thickness and the thermal properties of the coating material, it is possible to deduce the thickness profile within the range of view of the device as a field (specific to Coatmaster 3D technology vs. original Coatmaster technology) [14]. More complicated geometries than the plate substrate can also be handled as seen in the sample image for a spring illustrated in Figure 2. Here it can be observed that the thickness measured is greater at the outer portion of the spring, that is directly exposed to the particle cloud, than the inner portion of the spring. Which is an expected result as the inner portions are exposed to a more dispersed cloud after impact with the outer portions.
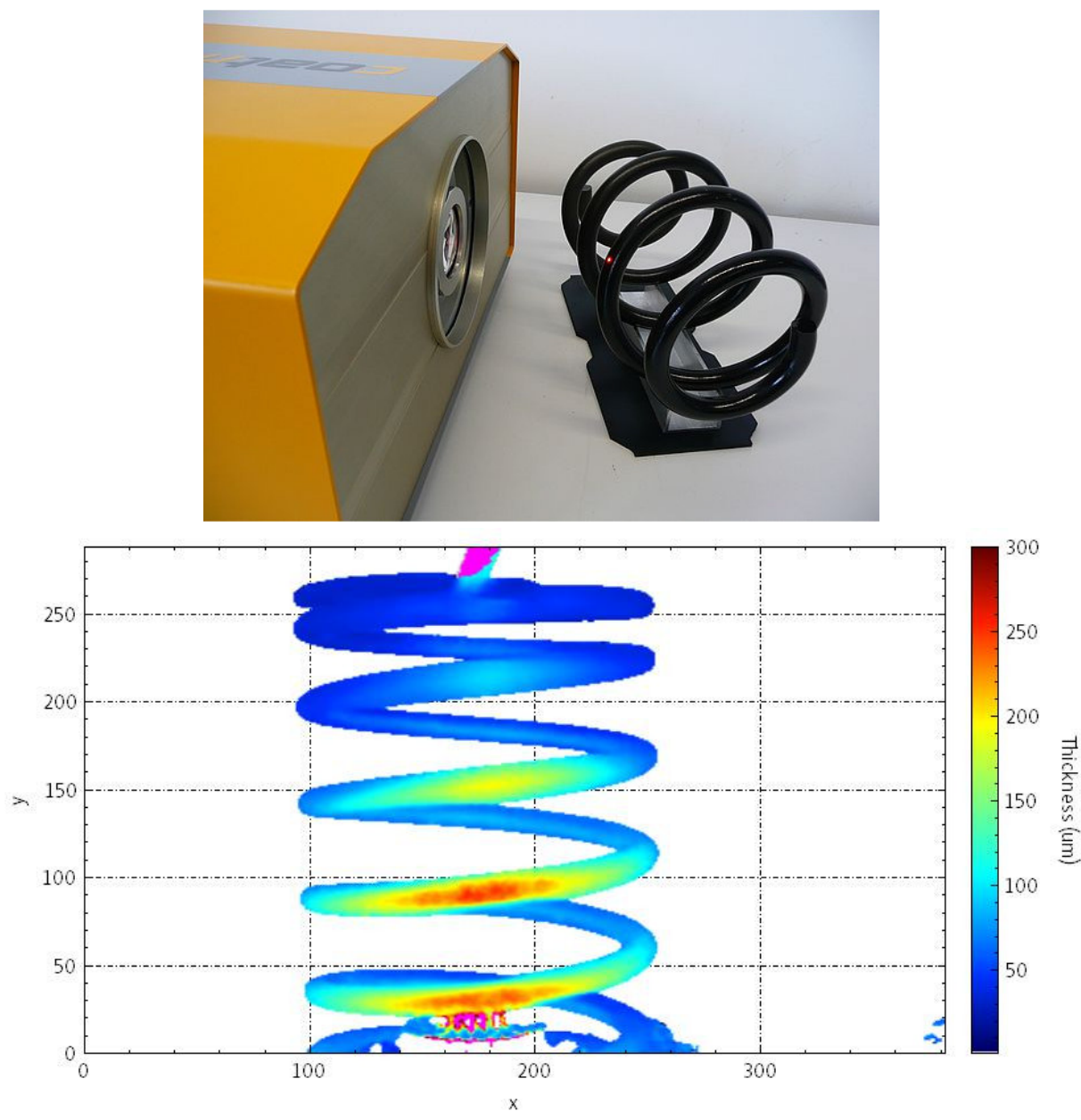

Figure 2- A sample image taken by Coatmaster [15] for a spring geometry and its in house quantification. 
Due to the dependency of the measurement on the coating material, as a first step, a calibration measurement must be performed on a cured specimen. For this purpose, a coating thickness gauge which can measure the thickness at a single point on the specimen can be employed. Once measurement with the gauge is obtained for a collection of points, covering the thickness range on the specimen, they are registered on the Coatmaster image which is then used as calibration data for the whole field. After the calibration process, any coating with that material within the thickness range of the specimen can be visualized and quantified.

\subsection{Assessment of the Measured Data}

Before assessing the data collected, the important characteristics of a coating procedure must be identified. First of these characteristics is the average thickness on the plate surface since it reflects the efficiency of the coating; ratio of the particles deposited on the plate to the total amount of particles, directly in the case of fixed amount of powder being utilized for each case. The second characteristic of interest is the center of the region of highest coating thickness which indicates where the thickest region of the coating achieved lies with respect to the geometrical center of the plate. The final characteristic derived relates to the uniformity of the coating. Significance of the latter lies in the fact that in many cases it is desired to have a homogeneous coating thickness on a specimen to provide uniform surface properties or functionality in practice.

\subsubsection{Data Filtering}

Prior to quantifying the essential characteristics for a coating, the data gathered from the Coatmaster must be filtered to eliminate noise. The noise in the data might be in form of pixels that either a) reach the upper threshold value specified during the calibration of the Coatmaster, b) obviously have very thin coating by inspection, or c) are islands of thickness values outside the substrate. In addition to the noise, geometric regions that correspond to clamps or grounding cables should be eliminated.

\subsubsection{Threshold Filtering}

The first step carried out in the filtering procedure is the elimination of the data that has a coating value outside a minimum and a maximum threshold. The minimum threshold can typically be slightly higher than zero as "noisy" pixels not on the substrate tend to contain small values. The maximum threshold should be set to a slightly lower value than the maximum set during the calibration of the Coatmaster, to eliminate pixels on the substrate that have a thin coating still exhibiting the threshold value as can be seen in the encircled area A in Figure 3 .

The effect of the threshold filtering is rather mild in the case depicted in Figure 3, as there are not large areas reaching the camera threshold value. However especially for the backside images of the substrate this is a necessary step due to the corner frame effect, causing large areas in the middle with thin coating displaying fictitious high values. 

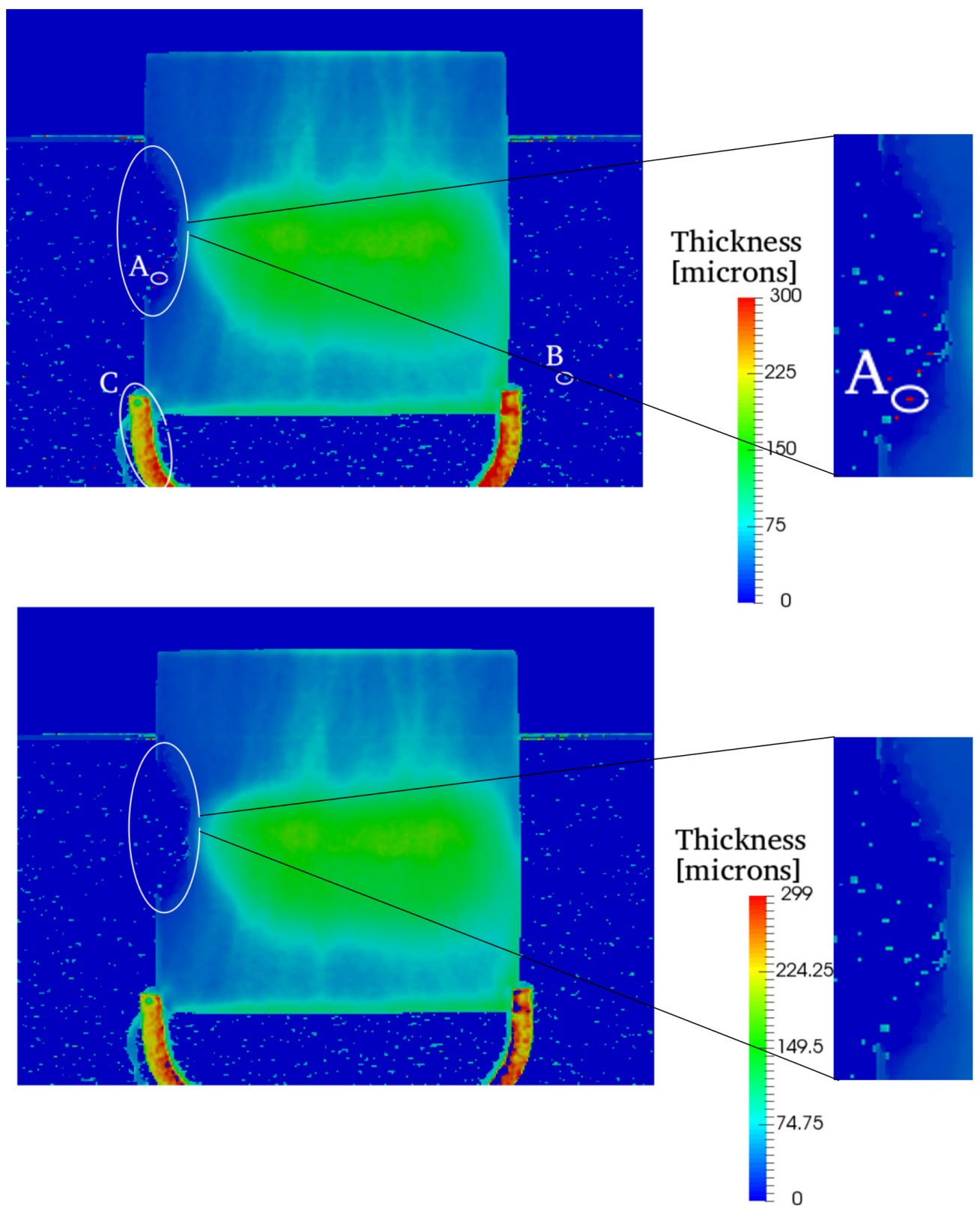

Figure 3- Threshold filtering of raw data image. On top is the raw image with pixels or regions marked as; A) a pixel island with thin coating reaching maximum threshold of the camera eliminated by threshold filtering; B) a pixel island with a thickness value outside the substrate eliminated by geometric filtering; C) clamp region eliminated with the corresponding filtering. Below is the image of the substrate after the threshold filtering with islands of type A eliminated. Scale fitted to data range available. 


\subsubsection{Geometric Filtering}

After the threshold filtering, not all the island pixels away from the substrate are necessarily eliminated as can be seen for the encircled pixel B in Figure 3. Since typically these pixels are small in comparison to the substrate a filtering based on geometric location can be carried out. To achieve this, the remaining unfiltered data is ordered based on its location in terms of rows and columns of an image. The row (r) and column (c) coordinates are depicted in Figure 5. Then on the distribution of the geometric locations, pixels with lower or higher location coordinates than specified limits defined in percentages, Фlow and $\Phi$ high, are eliminated as illustrated in Figure 4.

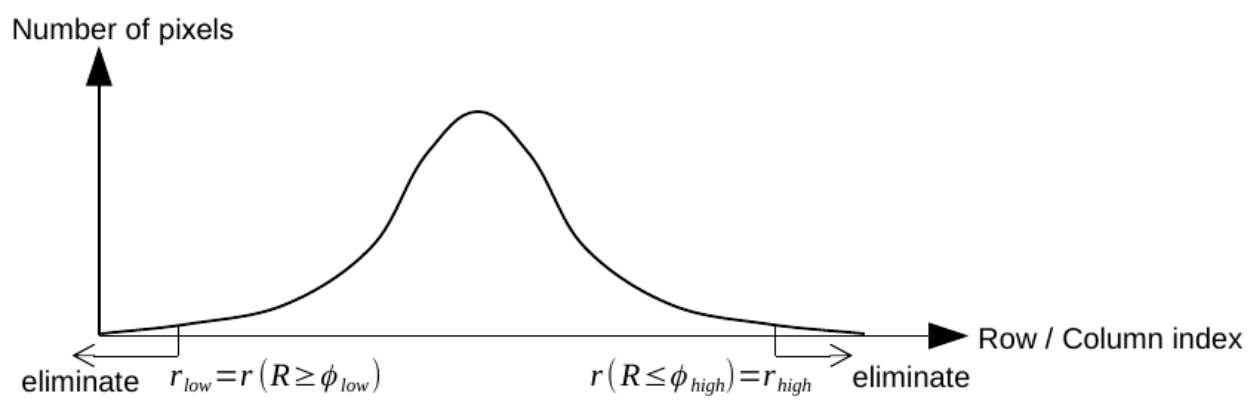

Figure 4- The geometric filtering based on the distribution function of geometric location.

This can be expressed mathematically with the aid of a filter delta function, defined in Equation (1), which multiplies the thickness value of a pixel i, $D_{i}$, that it is associated to.

$$
\begin{gathered}
D_{i}=D_{i} \cdot \delta_{i}^{\text {geo }} \\
\delta_{i}^{\text {geo }}=\begin{array}{c}
0 \Rightarrow r_{i}<r_{\text {low }} \vee r_{i}>r_{\text {high }} \vee c_{i}<c_{\text {low }} \vee c_{i}>c_{\text {high }} \\
1 \Rightarrow r_{\text {low }}<r_{i}<r_{\text {high }} \wedge c_{\text {low }}<c_{i}<c_{\text {high }}
\end{array}
\end{gathered}
$$

The effect of this filtering process can be seen in Figure 5 where the limits have been chosen as 2.5 and $97.5 \%$ of the coordinates for eliminating cells that contain a none zero coating thickness.

In this instance, it can be observed that not all the pixels that lie outside the substrate have been eliminated enclosed by the white box in Figure 5, most markedly on the bottom of the image. Also, a part of the clamp remains visible in the image. If the thresholding is carried out between $5-95 \%$ of the coordinate values, a better filtering is attained for the case under consideration as depicted in Figure 6. Hence the limiting threshold values are empirical. 

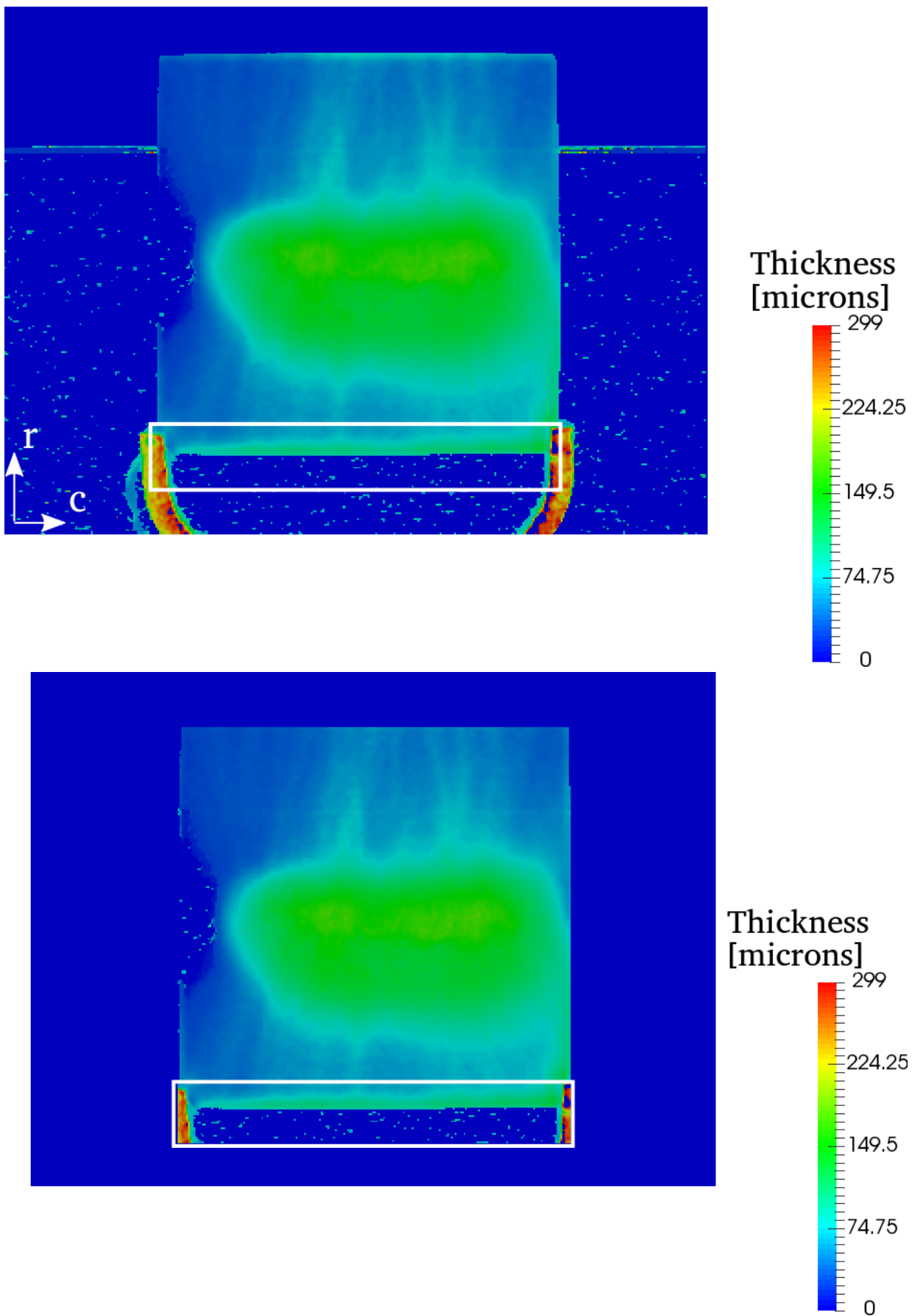

Figure 5- Geometric thresholding between 2.5-97.5\% of the pixel coordinates. On the top, the image of the substrate before, on the bottom, after the geometric filtering. Most pixels of type B in Figure 3 are eliminated, except those contained within the white box, as well as a portion of the region $\mathrm{C}$ in Figure 3 . Scale fitted to data range available. 

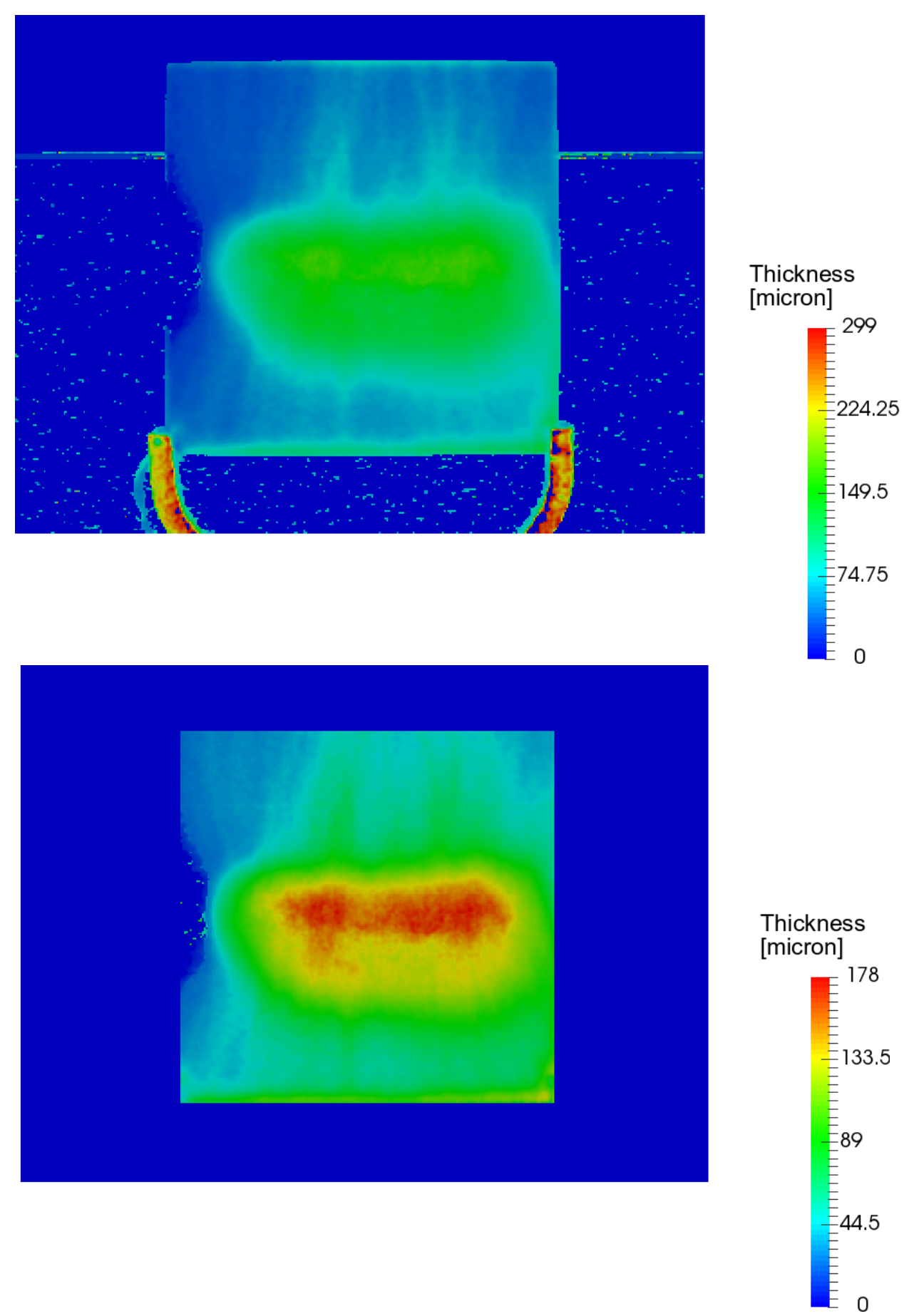

Figure 6- Geometric thresholding between 5-95\% of the pixel coordinates. On the top, the image of the substrate before, on the bottom, after the geometric filtering. Most pixels of type B in Figure 3 are eliminated as well as all of region $C$ in Figure 3. Scale fitted to data range available. 


\subsubsection{Clamp Region Elimination}

The clamps used to stabilize the substrate during the coating procedure usually exhibit high coating thicknesses. This region should be eliminated as it does not belong to the substrate. In addition, a portion of the coating in the vicinity on the substrate can be eliminated as they are resultant from the disturbance introduced by the clamp. The elimination of the clamp region is performed based on the schematic depicted in Figure 7, where the regions corresponding to the clamps are filled in blue. The identification of the plate center, as well as the extent of the plate in terms of row and column coordinates, play a central role in defining the clamp region.

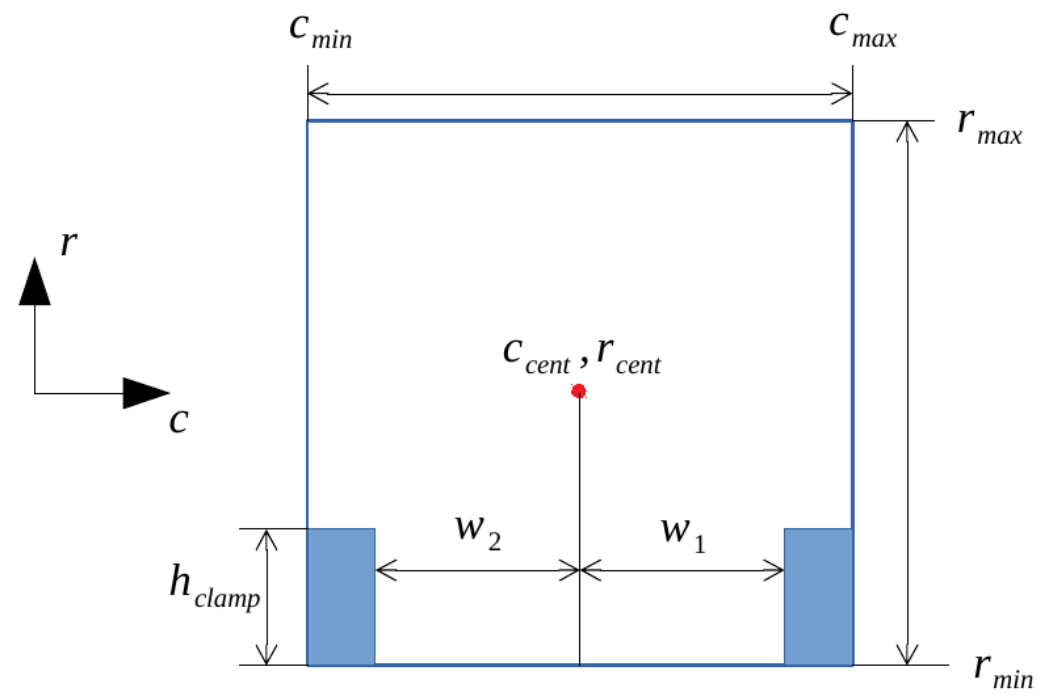

Figure 7- The clamp region schematic.

Two ratios govern the identification of the region as in Equation (2).

$$
\begin{gathered}
\frac{w_{1}}{c_{\text {max }}-c_{\text {cent }}}=\frac{w_{2}}{c_{\text {cent }}-c_{\min }}=f_{\text {width }} \quad \frac{h_{\text {clamp }}}{\max \left(r_{\max }-r_{\min }, c_{\max }-c_{\min }\right)}=f_{\text {height }} \\
r_{\text {pix }}<r_{\text {min }}+f_{\text {height }} \cdot \max \left(r_{\text {max }}-r_{\text {min }}, c_{\text {max }}-c_{\text {min }}\right) \wedge \\
\left(c_{\text {pix }}>c_{\text {cent }}+f_{\text {width }}\left(c_{\text {max }}-c_{\text {cent }}\right) \vee c_{\text {pix }}<c_{\text {cent }}-f_{\text {width }}\left(c_{\text {cent }}-c_{\text {min }}\right)\right)
\end{gathered}
$$

If the row coordinate is less than the minimum row plus the height of the clamp and the column coordinate is either less than that of the center plate minus the width w2 or greater than that of the plate center plus w1 then the data is to be eliminated as the clamp region. The effect of this filtering can be seen in Figure 8. For demonstrative purposes a larger area than the clamp has been eliminated, which may be useful in avoiding disturbances by the existence of the clamps. 

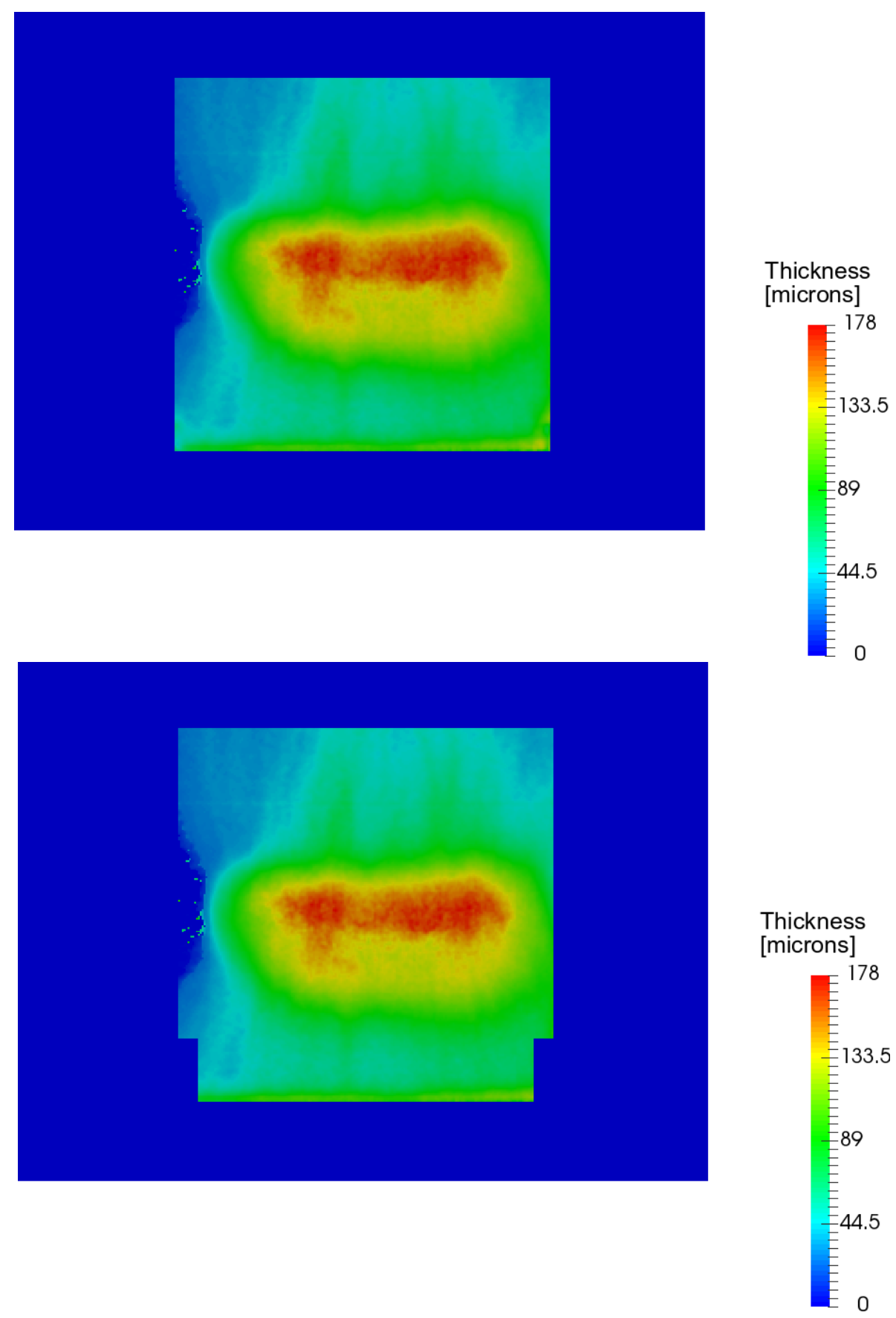

Figure 8- Clamp region filtering. 


\subsubsection{Average Coating Thickness (ACT)}

The first performance parameter that is essential in characterizing the quality of a coating is the average coating thickness. In the case of same amount of powder being utilized in process comparisons, it will give a direct indication of the efficiency as the substrate with the larger ACT will have deposited a larger proportion of the powder. Hence once the filtering procedure described in previous sections is applied, the ACT can be calculated by averaging the coating thickness from the remaining pixels as in Equation (4). The aim of any coating process is to have the highest possible efficiency hence the highest possible ACT in case of process parameter comparisons.

$$
\begin{gathered}
A C T=\frac{\sum_{i=1}^{N_{\text {unfilter }}} A_{i}}{A_{\text {plate }}} \frac{\sum_{i=1}^{N_{\text {unfilter }}} D_{i}}{N_{\text {unfilter }}} \\
A_{\text {plate }}=\left(r_{\text {max }}-r_{\text {min }}\right)\left(c_{\text {max }}-c_{\text {min }}\right)
\end{gathered}
$$

In Equation (4), the area factor is introduced especially for the cases with a thin coating, where the number of unfiltered cells, $N_{\text {unfilter }}$, is much less than the ones covering the whole plate. In these cases a direct comparison of the average thickness, obtained from area of individual unfiltered cells, $A_{i}$, would result in erroneous results if a scaling to the whole plate area is not performed. The plate area, $A_{\text {plate }}$, is obtained from the extent of the row and column coordinates. In comparisons between different measurements and plates, the area from the extent $A_{\text {plate }}$ is averaged over all the cases for the normalization, to eliminate the effect of variations, which are typically small for a plate substrate, for each case.

\subsubsection{Center Offset}

The next performance parameter is the center offset. Its quantification requires identification of a so-called region of highest coating thickness (RHCT). This is the region containing a specified percentage of the unfiltered cells that exhibit the highest coating thickness. The center offset is then calculated from the difference in row and column coordinates of the plate's geometric center (Equation (5)) and that of the region's (Equation (6)) as depicted in Figure 9.

$$
\begin{array}{r}
c_{\text {cent }}^{\text {geo }}=\frac{\sum_{i=1}^{N_{\text {unfilter }}} c_{i}}{N_{\text {unfilter }}} \quad r_{\text {cent }}^{\text {geo }}=\frac{\sum_{i=1}^{N_{\text {unfilter }}} r_{i}}{N_{\text {unfilter }}} \\
c_{\text {cent }}^{\text {reg }}=\frac{\sum_{i=1}^{N_{\text {unfilter }}} \delta_{i}^{\text {reg }} c_{i}}{\sum_{i=1}^{N_{\text {unfilter }}} \delta_{i}^{\text {reg }}} \quad r_{\text {cent }}^{\text {reg }}=\frac{\sum_{i=1}^{N_{\text {unfilter }}} \delta_{i}^{\text {reg }} r_{i}}{\sum_{i=1}^{N_{\text {unfilter }}} \delta_{i}^{\text {reg }}} \\
\delta_{i}^{\text {reg }}=\begin{array}{l}
0 \Rightarrow D_{i}<D_{\text {th }} \\
1 \Rightarrow D_{i} \geq D_{\text {th }}
\end{array}
\end{array}
$$

In Figure 9 the geometric center of the plate is shown in purple and that of the region in brown. A shift towards the bottom of the plate as well as to the right edge can be observed, indicating a certain asymmetry of the coating. Hence the center offset will be an indicator of the asymmetry of the coating. 

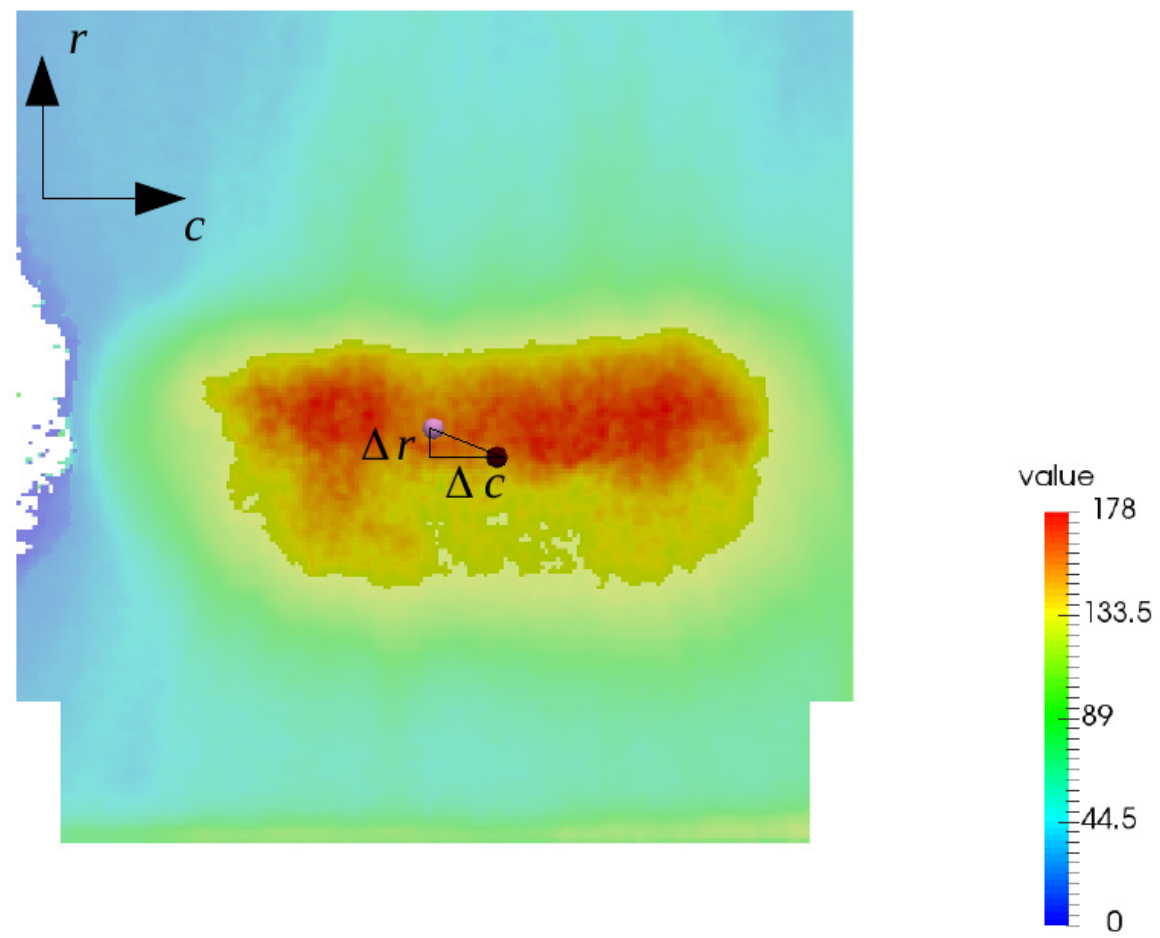

Figure 9- The geometric center of the plate versus the center of RHCT.

A center offset towards the bottom of the plate indicates that the substrate is placed far away from the pistol allowing gravity to be more effective, while an offset in the column direction usually indicates that the substrate is not necessarily oriented perpendicular to the pistol.

\subsubsection{Inhomogeneity}

The final performance parameter is the inhomogeneity. Its quantification is of the utmost importance whether it's desired to have an as homogeneous a coating as possible, or the exact opposite based on the application. The quantification of the parameter relies on the coating thickness histogram as illustrated in Figure 10.

To generate the coating thickness histogram, all the coating values are collected into a specified number of bins, in Figure 10, twenty bins are utilized. Hence each bar represents the number of pixels that have a thickness value contained within the extent of the bin. Out of this histogram the maximum number of counts, $N_{\max }$ and the corresponding average thickness value of that bin $D_{\max }$ are utilized to define the inhomogeneity, In accordance to Equation (7).

$$
\text { In }=\frac{\frac{1}{N_{b i n^{-1}}} \sqrt{\sum_{i=1}^{N_{\text {bin }}\left(D_{i}-D_{\max }\right)^{2} \frac{N_{i}}{N_{\max }}}}}{D_{\max }}
$$


Hence the inhomogeneity is some form of normalized, weighted standard deviation, where greater values of deviation indicate a less homogeneous coating distribution. The weight of number of counts in bin $i, N_{i}$, by the number of maximum counts ensures that a small number of pixels with large deviation from the maximum count thickness do not dominate the inhomogeneity value.

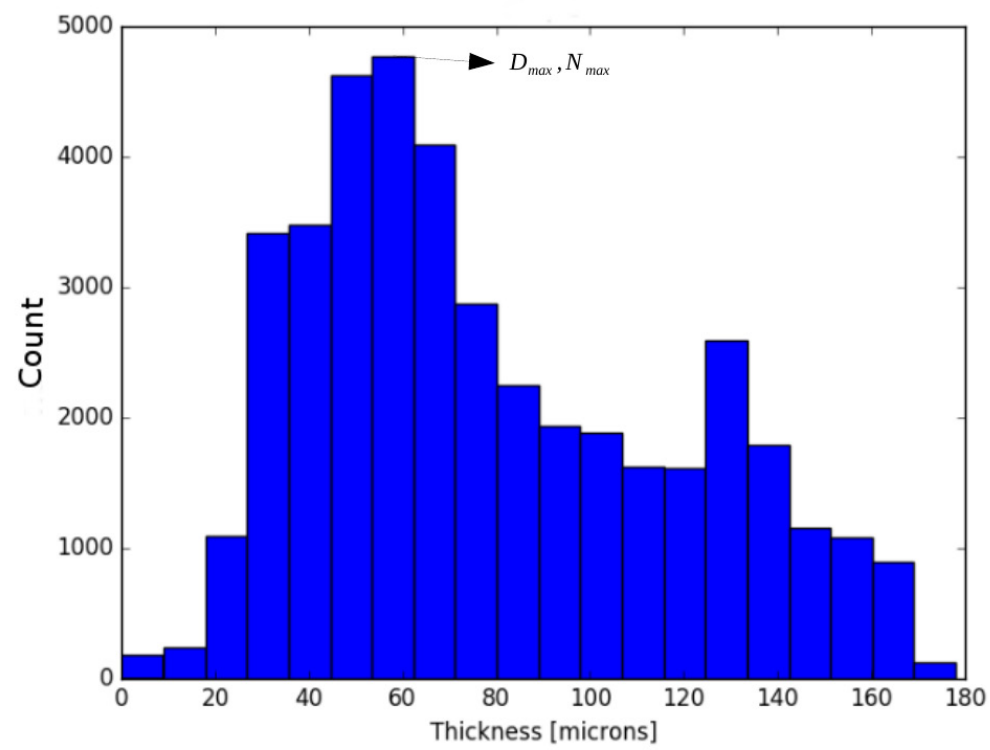

Figure 10- Thickness histogram of a coating.

\section{RESULTS \& VALIDATION}

The variation of the ACT versus the voltage can be observed in a box plot as illustrated in Figure 11. The data for the box plot comprises from that of three plates with each one having three measurements resulting in a total of 9 measurements as explained in Section 2.1. The ACT increases asymptotically and reaches a constant value at about $29 \mathrm{kV}$ applied in the pistol.

Even though the ACT levels out at higher voltages, the thickness contours for the median cases, depicted as red lines in the box plot, exhibit markedly different characteristics. Comparing the $24 \mathrm{kV}$ contour with the $52 \mathrm{kV}$ contour, it can be seen that a high coating thickness band at the center (B) and the bottom edge (C) along with a low coating thickness at the upper edge (A) of the plate can be observed in the former case, while the belt at the center diffuses (E) to confine the low coating thickness region to the corners (D) in the latter, resulting in a more homogeneous coating. This can also be verified quantitatively through the inhomogeneity plot in Figure 12. From the plot it can be observed that the inhomogeneity has initially a low value at the lowest voltage of $10 \mathrm{kV}$, increasing to a peak value at about $24 \mathrm{kV}$, then decreasing to attain its lowest values at the highest two voltages, which is quite consistent with the thickness contours depicted for the median cases.

The fact that the inhomogeneity at the lowest and highest voltages being at the same level can be explained if one considers the histogram plot of the coating thickness for three cases, namely at $10 \mathrm{kV}, 24 \mathrm{kV}$ and $52 \mathrm{kV}$ as illustrated in Figure 13. 

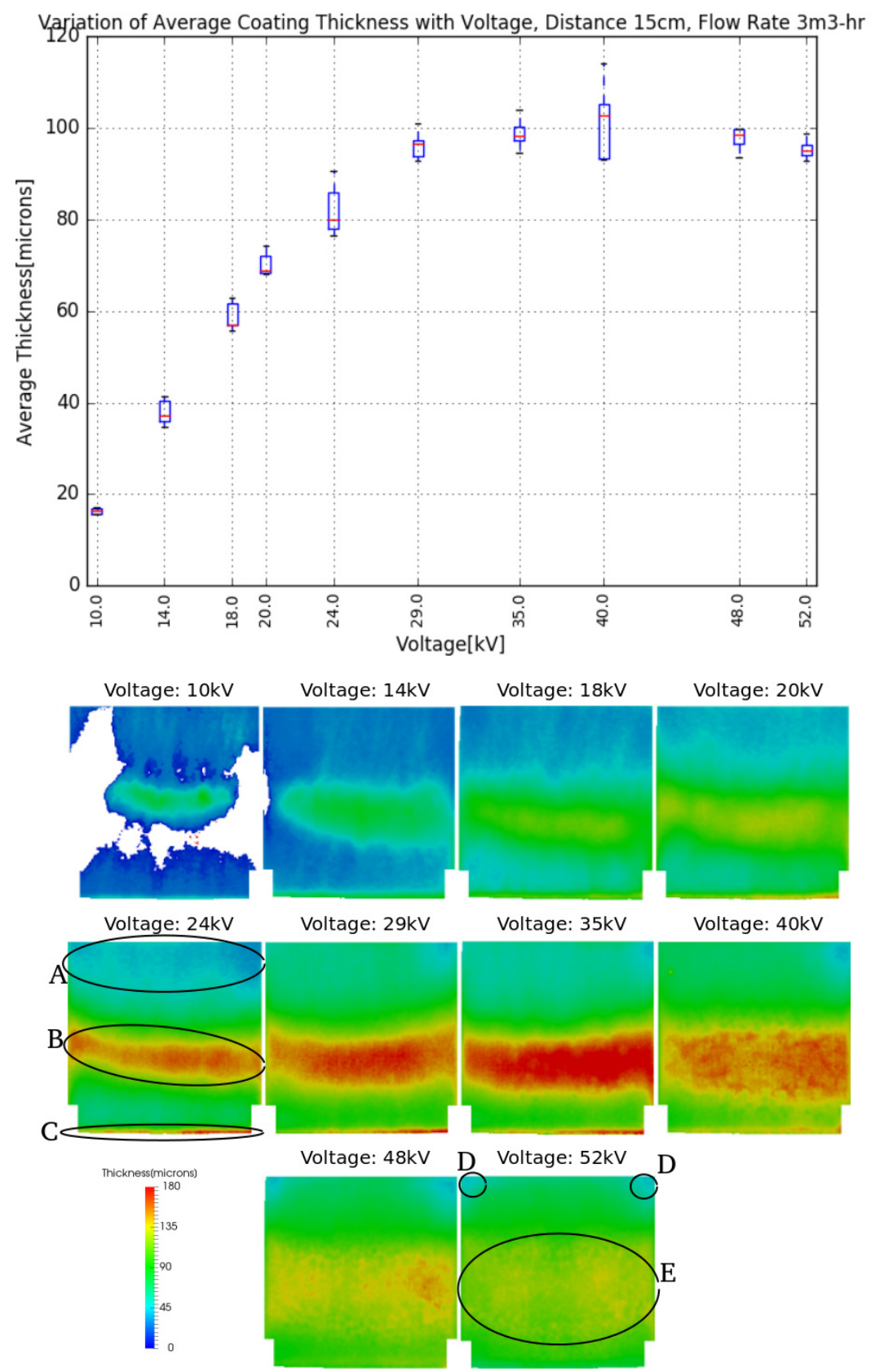

Figure 11- Average coating thickness vs voltage variation plot and thickness contours. On the bottom characteristic areas are marked as A) thin upper edge band, B) thick center band, C) bottom thick layer, D) thin corner confinements, E) diffused center band. 

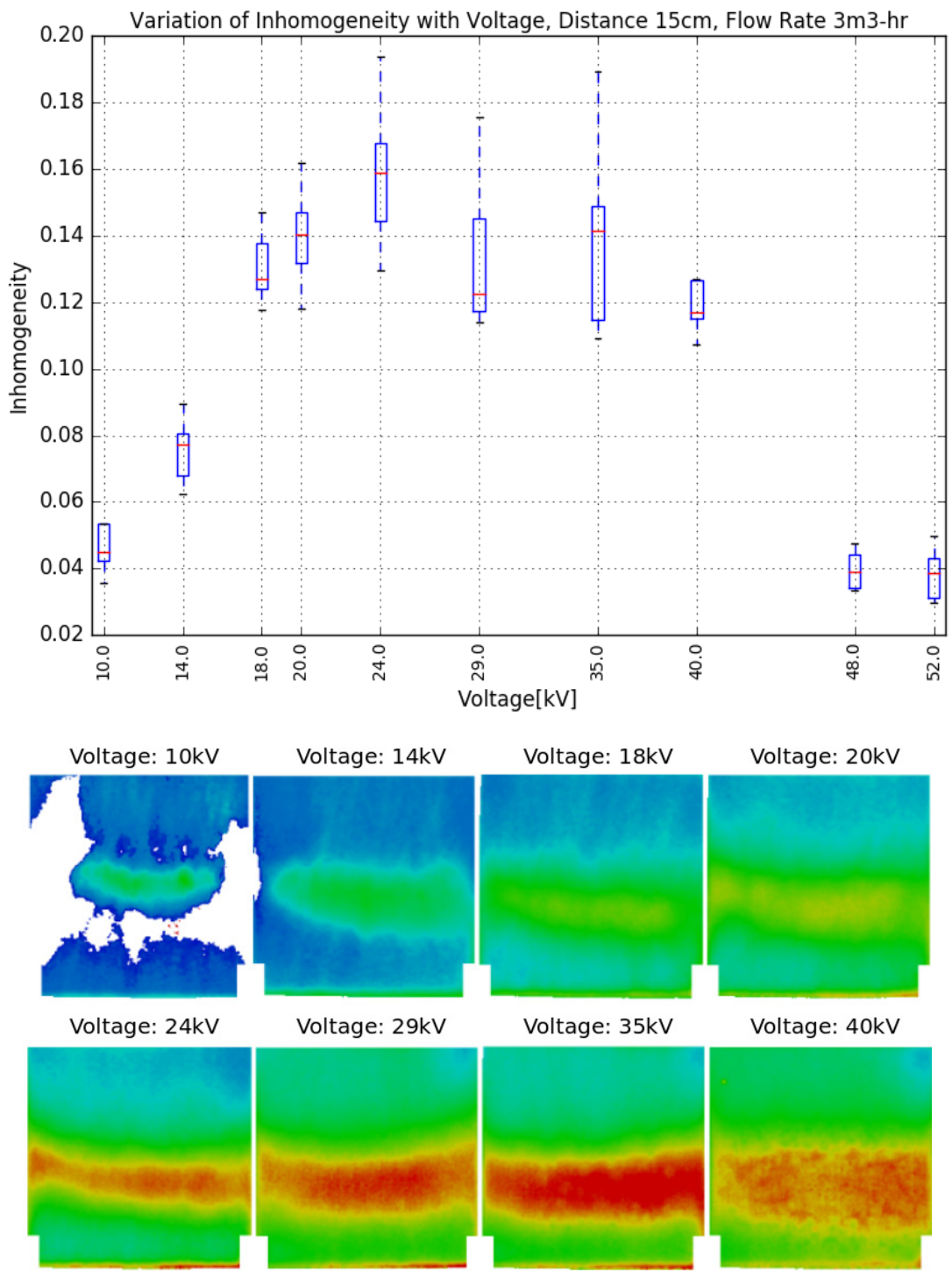

Voltage: $29 \mathrm{kV}$

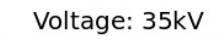

Voltage: $40 \mathrm{kV}$
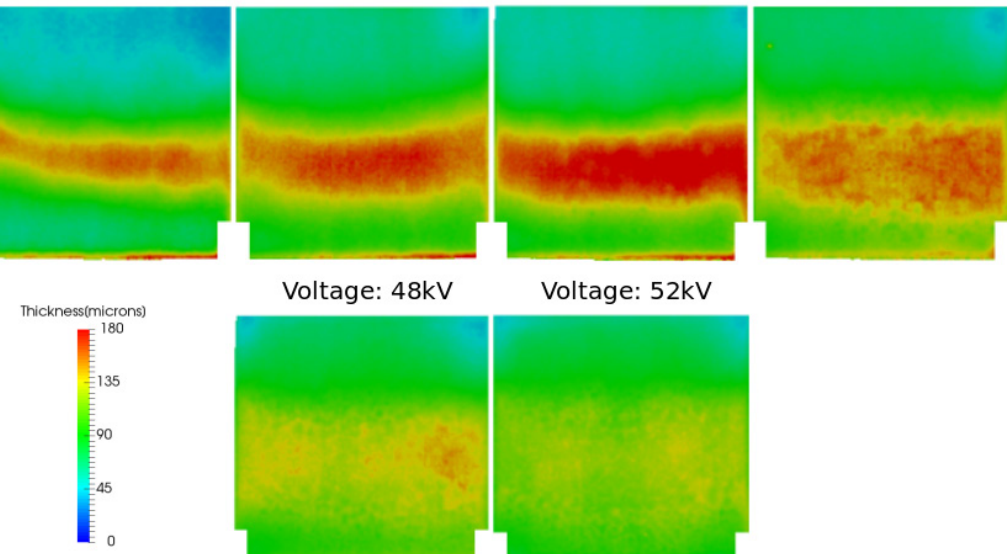

Voltage: $52 \mathrm{kV}$

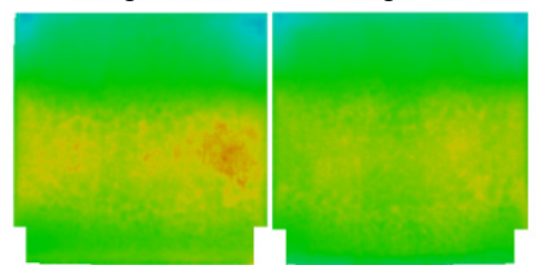

Figure 12- Inhomogeneity vs voltage variation plot and thickness contours. 

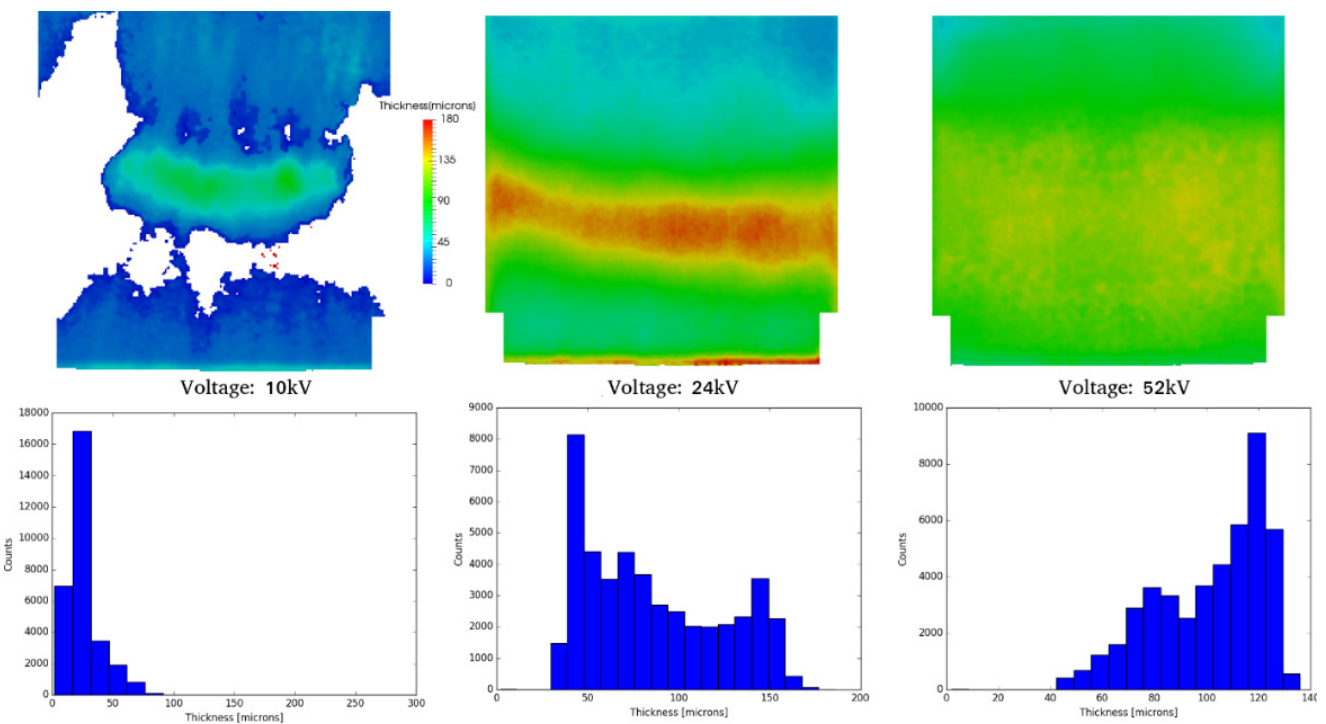

Figure 13- Histogram of coating thickness distribution for selected voltages.

The $10-\mathrm{kV}$ case has many pixels within a single bin which indicates that the inhomogeneity will be low. In fact, it would be expected that this case would exhibit a lower inhomogeneity than the $52-\mathrm{kV}$ case. However, the coating thickness associated with the highest count is a small value, hence the deviation from it is relatively quite large in the $10-\mathrm{kV}$ case. Whereas in the $52-\mathrm{kV}$ case this deviation is small, due to a large coating thickness being associated to the highest count, resulting in similar inhomogeneity values. For the intermediate voltage case, it can also be observed from the histogram that there is quite a large spread of coating thickness values with relatively large count numbers, the combination of which results in the peak value for the inhomogeneity.

The center offset is depicted for the row and the column coordinates on the plate substrate as in Figure 14. The negative values for the column coordinate indicate that the center of RHCT lies to the left of the plate center whereas a negative value for the row coordinate indicates that it lies below the plate center. It can be observed that all the median values for the column coordinates are positive seemingly without a clear trend while they are negative for the row coordinates, approaching the plate center with increasing voltage.

The three selected cases for the column coordinate namely the $18,35,52 \mathrm{kV}$ on the left side of Figure 14 depict an almost aligned column coordinate with the center of the plate at $18 \mathrm{kV}$, then a larger offset at $35 \mathrm{kV}$ increasing even more at $52 \mathrm{kV}$, serving as a visual validation of the center offset vs the voltage plot. Similarly, on the right-hand side, it can be observed that the row coordinate approaches the plate center with increasing voltage, from 20 to 29 and $48 \mathrm{kV}$, as reflected in the row coordinate center offset plot. It should be noted that especially at intermediate voltage levels, there is typically a thickly coated region at the bottom edge of the plate (see area C in Figure 11) which pushes the RHCT center towards the bottom, most evident for the case of $20 \mathrm{kV}$.

The center offset can also provide important information on the coating procedure. In Figure 14, it is observed that all the median values for the center offset of the column coordinate as well as most part of the range boxes are all in the positive value range, meaning that the offset is systematically towards the right side of the plate center, since otherwise it 

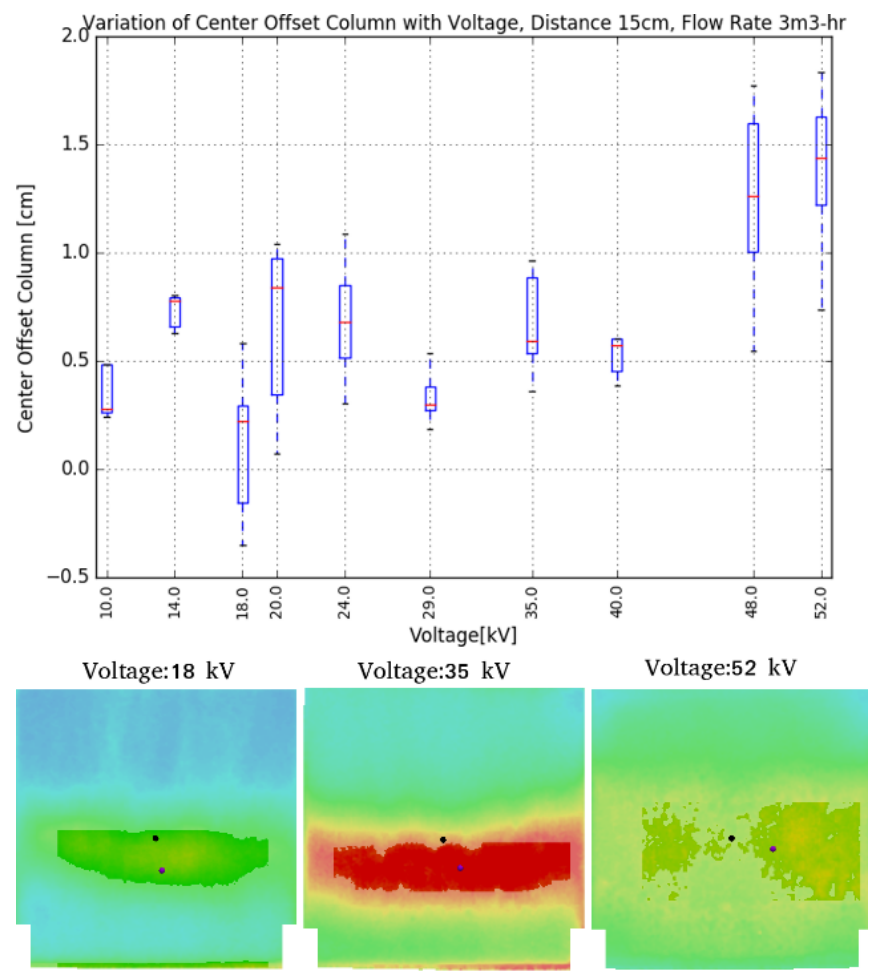

Thickness(microns)
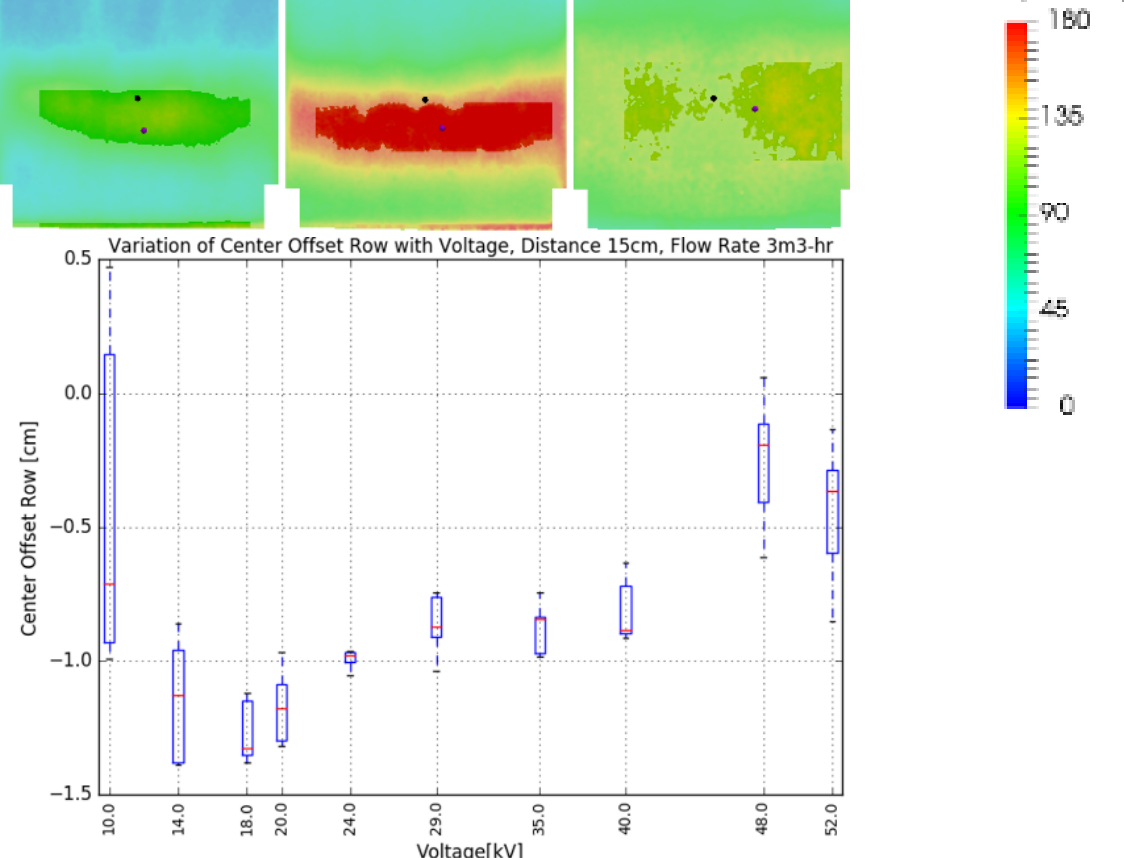

Voltage: $20 \mathrm{kV}$

Voltage: $29 \mathrm{kV}$

Voltage:48 $\mathrm{kV}$
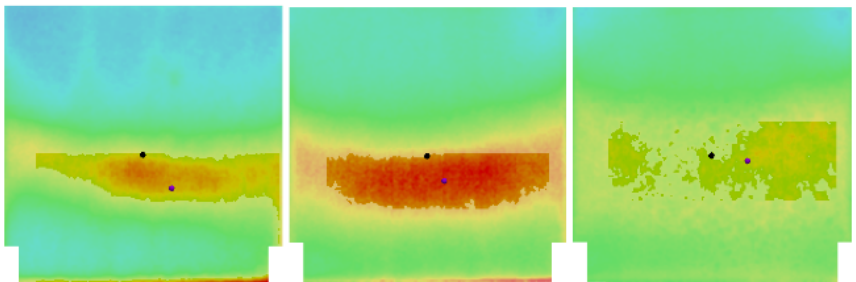

Figure 14- Variation of center offset in column and row coordinates versus the voltage. 
would be expected that the range boxes would encompass an offset value of 0 . A likely cause of this systematic effect could be the ventilation in this case. When the offset for the row coordinate is considered, except for the very low voltage of $10 \mathrm{kV}$, all the offsets are systematically below the plate center, approaching it with increasing voltage. This is an expected result since the systematic effect here is gravity, the effect of which decreases as the electrostatic forces become dominant due to higher voltages, pushing the center of the RHCT towards the plate center.

\section{SUMMARY \& CONCLUSION}

In this paper, the procedure to gather and treat experimental data pertaining to powder coating processes is presented in the form of the novel method of gathering the coating thickness data from the advanced thermal optics technology based Coatmaster and its filtering. A key aspect of this study is the performance parameters derived for quantification of the quality of a coating. All these three aspects form the basis for an extensive numerical solver validation, which subsequently will be a key element in optimizing process parameters; voltages, flow rates, and geometric orientation of the substrate with respect to the pistol, for given performance criteria in terms of the ACT and inhomogeneity.

The importance of the performance parameters derived and their intercorrelation is well established in this study. To that effect, one might be tempted to assess the quality of a coating only through the ACT values, which would lead to the conclusion that voltages higher than about $29 \mathrm{kV}$, where the asymptotic limit is reached as observed in Figure 11, do not affect the quality of the coating. However, this conclusion is erroneous considering the inhomogeneity of the coating. As depicted in Figure 12, the coating becomes homogeneous only for the highest two voltages applied.

Hence while assessing the quality of a coating procedure, the homogeneity of the coating should be considered alongside the ACT, as for virtually the same ACT values homogeneity of the coating can be quite different. From the ACT and inhomogeneity versus voltage graphs illustrated in Figure 11 and Figure 12 respectively, it would be possible to deduce ideal voltage ranges based on the application. For example, a process requiring a thin uniform coating should remain at low voltage values of around $10-14 \mathrm{kV}$, whereas voltage values above up to $24 \mathrm{kV}$ will produce thin coatings on average with a thicker concentration in the middle of the plate. The same distribution but associated to a larger ACT can be attained in the range from $29-40 \mathrm{kV}$, thereafter the coating becoming more uniform with increasing voltages.

The other performance parameter, the center offset of the RHCT, will be informative for the symmetry of the coating thickness and possibly be an indicator of systematic secondary effects. In this study, for the plate substrate, a systematic deviation from symmetry emerged as a result of the assessment of this parameter, where the left side of the plates were coated thinner compared to the right side. This is attributed to a secondary effect, most probably to the air flow driven by the ventilation system. Furthermore, this parameter is also an indirect but sound indication of how strong the electrostatic forces are in comparison to the gravitational ones, as larger offsets towards the bottom of the plate imply weaker electrostatic forces. This would be a valuable information especially in optimizing the distance between the substrate and the pistol.

The filtering methods described here for the planar substrate are applicable for any convex geometry stemming from the Coatmaster measurement, which provides a two-dimensional data set. In order to generalize the method to handle complex geometries that are frequently 
encountered in practice, the procedure requires an extension to handle islands of noisy data that may reside in a concave part of a given substrate. Common image processing techniques such as median and Gaussian filters [16] will be employed as further development of the methods described to address this issue.

\section{REFERENCES}

[1] Boiger G, Euler-Lagrangian Model of Particle Motion and Deposition Effects in Electro-Static Fields Based on OpenFoam, The International Journal of Multiphysics, 2016, 10(2), 177-194.

[2] Bariska A, Reinke N, Berührungslose thermische Schichtprüfung, School of Engineering, Zurich University of Applied Sciences (ZHAW), Winterthur, Switzerland. Swiss Engineering STZ, 2011, 57.16.

[3] Winterthur Instruments, Operator Manual CoatMaster, 2017.

[4] Pietschmann, Judith. Industrielle Pulverbeschichtung: Grundlagen,Verfahren, Praxiseinsatz. 4th ed. JOT-Fachbuch. Springer Vieweg, 2013.

[5] Pulli, Karlheinz. Optimierung der Pulverlackapplikation durch Anwendung experimenteller und numerischer Untersuchungsverfahren, 2006.

[6] Wang, Fujing, Robert Martinuzzi, and Jesse (Jing-Xu) Zhu, Experimental Study of Particle Trajectory in Electrostatics Powder Coating Process, Powder Technology, 2005,150(1), 20-29.

[7] Thomas, Aline, Khashayar Saleh, Pierre Guigon, and Claire Czechowski, Characterisation of Electrostatic Properties of Powder Coatings in Relation with Their Industrial Application, Powder Technology, 2009,190(1-2), 230-235.

[8] Dastoori, K, and B Makin, Adhesion Measurements for Electrostatic Powder Coatings Using Drop Test Rig and Virtual Oscilloscope, Journal of Electrostatics, Electrostatics 2001: 9th International Conference on Electrostatics, 2001, 51-52, 509-514.

[9] Dastoori, K., B. Makin, and J. Telford, Measurements of Thickness and Adhesive Properties of Electrostatic Powder Coatings for Standard and Modified Powder Coating Guns, Journal of Electrostatics, Electrostatics 2001: 9th International Conference on Electrostatics, 2001, 51-52, 545-551.

[10] Meng, Xiangbo, Jingxu (Jesse) Zhu, and Hui Zhang, Influences of Different Powders on the Characteristics of Particle Charging and Deposition in Powder Coating Processes, Journal of Electrostatics, 2009, 67(4), 663-671.

[11] Shah, U., J. Zhu, C. Zhang, and J.H. Nother, Numerical Investigation of Coarse Powder and Air Flow in an Electrostatic Powder Coating Process, Powder Technology, 2006, 164(1), 22-32.

[12] Böttner, C.-U, and M Sommerfeld, Numerical Calculation of Electrostatic Powder Painting Using the Euler/Lagrange Approach, Powder Technology, 2002, 125(2-3), 206-216.

[13] Ye, Q., T. Steigleder, A. Scheibe, and J. Domnick, Numerical Simulation of the Electrostatic Powder Coating Process with a Corona Spray Gun, Journal of Electrostatics, 2002, 54(2), 189-205. 
[14] https://coatmaster.ch/en/coatmaster/functionality/

[15] https://coatmaster.ch/en/coatmaster/products/erweiterungen/1-point-marker/

[16] Burger W, Burge M., Principles of digital image processing: core algorithms, London: Springer; 2009. 327 p.

[17] Boiger G., Characterization of Particle Motion and Deposition Behaviour in ElectroStatic Fields, The International Journal of Multiphysics, 10(2), 195-204. 